\title{
Five and 10 minute Apgar scores and risks of cerebral palsy and epilepsy: population based cohort study in Sweden
}

\author{
Martina Persson, ${ }^{1}$ Neda Razaz, ${ }^{1}$ Kristina Tedroff, ${ }^{2}$ KS Joseph, ${ }^{3}$ Sven Cnattingius ${ }^{1}$
}

${ }^{1}$ Department of Medicine,

Solna, Clinical Epidemiology

Unit, Karolinska Institutet,

SE-171 76 Stockholm, Sweden

2Department of Women's and Children's Health,

Neuropediatric Unit, Karolinska Institutet, Stockholm, Sweden

${ }^{3}$ Department of Obstetrics and Gynaecology, School of

Population and Public Health, University of British Columbia and the Children's and

Women's Hospital of British Columbia

Correspondence to: M Persson Martina.Persson@ki.se

Additional material is published online only. To view please visit the journal online.

Cite this as: BMJ 2018;360:k207 http://dx.doi.org/10.1136/bmj.k207

Accepted: 3 January 2018

\section{ABSTRACT}

OBJECTIVE

To investigate associations between Apgar score at five and 10 minutes across the entire range of score values (from 0 to 10) and risks of childhood cerebral palsy or epilepsy, and to analyse the effect of changes in Apgar scores from five to 10 minutes after birth in infants born $\geq 37$ completed weeks.

\section{DESIGN, SETTING, AND PARTICIPANTS}

Population based cohort study in Sweden, including 1213470 non-malformed live singleton infants, born at term between 1999 and 2012. Data on maternal and pregnancy characteristics and diagnoses of cerebral palsy and epilepsy were obtained by individual record linkages of nationwide Swedish registries.

\section{EXPOSURES}

Apgar scores at five and 10 minutes.

\section{MAIN OUTCOME MEASURE}

Cerebral palsy and epilepsy diagnosed up to 16 years of age. Adjusted hazard ratios were calculated, along with $95 \%$ confidence intervals.

RESULTS

$1221(0.1 \%)$ children were diagnosed as having cerebral palsy and 3975 (0.3\%) as having epilepsy. Compared with children with an Apgar score of 10 at five minutes, the adjusted hazard ratio for cerebral palsy increased steadily with decreasing Apgar score: from 1.9 (95\% confidence interval 1.6 to 2.2 ) for an Apgar score of 9 to 277.7 (154.4 to 499.5) for an Apgar score of 0 . Similar and even stronger associations were obtained between Apgar scores at 10 minutes and cerebral palsy. Associations between Apgar scores and epilepsy were less pronounced, but increased hazard ratios were noted in infants with a five minute Apgar score of 7 or less and a 10 minute Apgar score of 8 or less. Compared with infants with an Apgar of 9-10 at both five and 10 minutes, hazard ratios of cerebral palsy and epilepsy were higher among infants with a five minute Apgar score of 7-8 and a 10 minute Apgar score of 9-10.

\section{WHAT IS ALREADY KNOWN ON THIS TOPIC}

A low Apgar score (0-3 or 4-6) at five minutes after birth increases risks of cerebral palsy and epilepsy

\section{WHAT THIS STUDY ADDS}

Risks of cerebral palsy and generally also epilepsy increase with decreasing Apgar scores at five and 10 minutes

A reduced Apgar score at 10 minutes confers higher risks of cerebral palsy and epilepsy than does a reduced Apgar score at five minutes

Slight changes within the normal Apgar score range (7-10) from five to 10 minutes also influence risks of cerebral palsy and epilepsy

\section{CONCLUSION}

Risks of cerebral palsy and epilepsy are inversely associated with five minute and 10 minute Apgar scores across the entire range of Apgar scores.

\section{Introduction}

The Apgar score is a vitality index from 0 to 10 assigned to virtually every newborn infant at one, five, and 10 minutes after birth. The score is based on measures of heart rate, respiratory effort, skin colour, muscle tone, and reflex irritability. A total score of 7-10 is considered "normal," and a lower Apgar score indicates depressed vitality. ${ }^{1}$ However, several possible causes of low Apgar scores exist, such as perinatal asphyxia, congenital infections, maternal fever in labour, a diagnosis of chorioamnionitis, malformations, and preterm birth..$^{2-8}$

Population based studies have shown that risks of cerebral palsy and epilepsy are increased in children with low Apgar scores, and a low Apgar score at five minutes confers a higher risk than a correspondingly low Apgar score at one minute. ${ }^{9-12}$ We are aware of only one previous study investigating risks associated with a low 10 minute Apgar score. This study showed that the risk of developing cerebral palsy was significantly higher in children with a 10 minute Apgar score between 0 and 3 compared with children who had a similar score at five minutes. ${ }^{13}$ No previous study has investigated risks of neurological disorders in children with more modestly depressed Apgar scores of 4-6 at 10 minutes or the risks of childhood neurological disorders across the full range of Apgar scores (that is, at each score value from 0 to 10).

Changes in Apgar score values between one and five minutes are known to influence risks of cerebral palsy and epilepsy. Children with a low Apgar score of 0-3 at one minute and a normal score of 7-10 at five minutes have substantially higher risks of cerebral palsy and epilepsy compared with those who have normal Apgar scores (between 7 and 10) at both one and five minutes. ${ }^{1011}$ Although neurological morbidity may be influenced by changes in Apgar scores between five and 10 minutes, we are unaware of any study focusing on this question. If modest changes in normal Apgar scores (that is, changes within the range of 7 to 10) from five to 10 minutes influence risks, this would provide justification for continuing resuscitation of infants who have not attained an Apgar score of 10 at five minutes.

In this population based study, we investigated the associations between Apgar scores at five and 10 minutes and risks of cerebral palsy and epilepsy in singleton infants born at term ( $\geq 37$ weeks). We were particularly interested in examining the effect of a change in Apgar scores from five to 10 minutes, including changes within the normal range of Apgar 
scores. We hypothesised that risks of cerebral palsy and epilepsy would increase with decreasing Apgar scores, in particular at 10 minutes. We further hypothesised that even modestly depressed Apgar scores as well as minor changes between the five and 10 minute Apgar scores would influence risks of cerebral palsy and epilepsy.

\section{Methods}

The source population included all live births in Sweden with individual level information obtained from several Swedish national registries linked using the unique national registration numbers of mothers and their liveborn offspring. ${ }^{14}$ National registries used included the Medical Birth Register (MBR), ${ }^{15}$ which contains information on antenatal, obstetric, and neonatal care that is prospectively recorded on standardised forms for more than $98 \%$ of all births in Sweden. Another registry, the nationwide National Patient Register, ${ }^{16}{ }^{17}$ has included diagnostic codes on hospital inpatient care since 1987 and hospital outpatient care from 2001. Diagnoses in these databases were coded using the Swedish versions of the international classification of diseases, ninth revision (ICD-9), from 1987 to 1996 and ICD-10 from 1997 onwards. Information on maternal education and country of origin came from the Education Register and the Total Population Register, respectively. ${ }^{1418}$

\section{Study population}

Between 1999 and 2012, the MBR contained information on 1379482 live singleton infants. After exclusion of preterm infants ( $<37$ completed weeks, $\mathrm{n}=73101$ ), infants with congenital malformations ( $\mathrm{n}=70$ 615), and records with missing data on maternal or child identification numbers ( $\mathrm{n}=15998)$, the study cohort included 1219768 live singleton term infants. Complete information on Apgar scores recorded at one and five minutes was available for 1213470 (99\%) infants. Among infants with complete information on Apgar scores at one and five minutes, information on Apgar score at 10 minutes was available for 1211733 (99.9\%).

\section{Definition of outcome}

Identification of cases of cerebral palsy was based on the presence of one or more diagnostic codes for cerebral palsy (ICD-9 code 343; ICD-10 code G80), recorded between 1999 and 2012. Epilepsy in children was identified as follows ${ }^{1920}$ : an occurrence of at least two diagnostic codes for epilepsy (ICD-9 code 345; ICD10 code G40) on separate dates; or an occurrence of at least one diagnostic code for convulsions (ICD-9 code 780.3; ICD-10 code R56) and at least one diagnostic code for epilepsy, in separate medical encounters; the diagnosis of convulsions had to precede that of epilepsy. Diagnostic codes for cerebral palsy in all medical records were eligible for consideration. However, we restricted the diagnosis of epilepsy to the period after the child's 27th day after birth, to avoid misclassifying neonatal convulsions as epilepsy. Only children with epilepsy who did not also have cerebral palsy were retained in the study $(n=371$ infants with epilepsy and cerebral palsy as a comorbidity were excluded). We considered the date associated with the first record of epilepsy or cerebral palsy to be the date of diagnosis, up to 16 years of age.

\section{Main exposures}

Apgar scores at five and 10 minutes were the main exposures. We analysed Apgar scores in several ways: revised categories (Apgar values of 0-2, 3-4, 5-6, 7-8, and 9-10) and each score value (Apgar values of 0,1 , 2, 3, 4, 5, 6, 7, 8, 9, and 10).

\section{Other covariates}

Maternal characteristics of interest included age at delivery, country of origin, highest attained level of education, cohabitation with a partner, parity, height, body mass index, smoking during pregnancy, mode of delivery, and maternal epilepsy. We calculated maternal age at delivery as the date of delivery minus the mother's birth date. We defined birth order (parity) as the number of births to each mother. We calculated body mass index $\left(\mathrm{kg} / \mathrm{m}^{2}\right)$ by using weight (wearing light indoor clothing) measured at registration to antenatal care and self reported height and categorised it according to the World Health Organization classification as underweight $(<18.5)$, normal weight (18.5 to $<25)$, overweight $(25$ to $<30)$, obesity grade I (30 to $<35$ ), obesity grade II (35 to $<40)$, or obesity grade III $(\geq 40) .^{21}$ The cohabitation status of the mother was obtained at the first antenatal visit. Mothers who reported daily smoking at the first antenatal visit and/or at 30-32 gestational weeks were classified as smokers, whereas mothers who stated that they did not smoke at either time point were classified as non-smokers. We defined maternal epilepsy before the child's birth by using the same algorithm as for children. The covariate categories used are listed in supplementary table A.

In Sweden, all women are offered an ultrasound scan at 18 gestational weeks or earlier for dating and screening for abnormalities. We estimated gestational age (in completed weeks) by using the following hierarchy: the date of early second trimester ultrasound scan (87.7\%), the date of the last menstrual period (7.4\%), or a postnatal assessment (4.9\%). We categorised birth weight for gestational age as small ( $<10$ th centile), appropriate (10th-90th centile), or large ( $>90$ th centile) for gestational age and sex, using the current Swedish standard for normal fetal growth. ${ }^{22}$

\section{Patient involvement}

No patients were involved in setting the research question or the outcome measures, nor were they involved in developing plans for or implementation of the study. No patients were asked to advise on interpretation or writing up of results. There are no plans to disseminate the results of the research to study participants or the relevant patient community. 


\section{Statistical analyses}

Because the duration of follow-up differed between study participants born between 1999 and 2012, we estimated person time incidence rates of epilepsy and cerebral palsy. For cerebral palsy, children were followed from birth until the date of the first diagnosis of cerebral palsy, emigration, death, 16 years of age, or the end of follow-up (31 December 2012), whichever came first. For epilepsy, we followed each child from the 28th day after birth until the date of the first diagnosis of epilepsy, emigration, death, or end of follow-up (31 December 2012), whichever occurred first. We used Cox proportional hazard regression to estimate hazard ratios with 95\% confidence intervals. Cox regression, which is a multivariable survival analysis model, accounts for varying duration of follow-up between study participants-that is, the varying duration from birth to date of diagnosis or end of follow-up. We specified the robust sandwich estimate of the covariance matrix to account for the correlations of sequential births to the same woman in the study. We used multivariable Cox regression analysis to compare the rates of epilepsy and cerebral palsy between children with differing Apgar score values at five and 10 minutes. Confounders included in the final models were based on the literature or statistical significance $(\mathrm{P}<0.10){ }^{23-27}$ The full model included maternal factors (maternal age, country of origin, education level, and smoking) and birth characteristics of the child (birth order, gestational age (in days), birth weight for gestational age, and year of birth) (see supplementary table A). In analyses of epilepsy, we also adjusted rates for maternal epilepsy. Information on maternal body mass index was missing in $10.6 \%$ of all pregnancies, owing to missing values of maternal height, weight, or both. We therefore adjusted for maternal height and body mass index only in supplementary analyses. We assessed a linear trend in the association between Apgar scores at five and 10 minutes and offspring's epilepsy or cerebral palsy by introducing a variable representing the ordinal categories of the Apgar score as a continuous predictor into the model. We used two sided $\mathrm{P}$ values of less than 0.05 to indicate statistical significance. We used the SAS software package 9.4 for all analyses.

\section{Results}

Maternal and infant characteristics and rates of low Apgar scores

Rates of low (0-3 or 4-6) Apgar scores at five minutes in the study population generally increased with increasing maternal body mass index and decreasing height. Rates of low Apgar scores at five minutes were also higher in the offspring of non-cohabiting mothers but were not substantially influenced by maternal age, education, smoking, country of birth, or year of delivery. Apgar scores of 0-3 at five minutes were equally common in offspring of mothers with and without epilepsy, but scores of 4-6 were more common in offspring of mothers with epilepsy. Rates of low Apgar scores differed by mode of delivery, and the highest rates were recorded in offspring delivered by emergency caesarean section. With respect to gestational age, the highest rate of low Apgar scores was observed in post-term infants ( $\geq 42$ weeks); rates of low Apgar scores were higher in small for gestational age infants compared with offspring born at appropriate weight for gestational age and in boys compared with girls (supplementary table A).

\section{Apgar scores at five and 10 minutes and risks of cerebral palsy and epilepsy}

In the study cohort, $1221(0.1 \%)$ children were diagnosed as having cerebral palsy, corresponding to an incidence rate of 1.5/10 000 child years. Compared with children with an Apgar score of 10 at five minutes, children with lower Apgar scores had increased hazard ratios of cerebral palsy (fig 1). Hazard ratios of cerebral palsy consistently increased with decreasing Apgar score values: from 1.9 in children with an Apgar score of 9 at five minutes to 277.7 in those with an Apgar score of 0 at five minutes. Low Apgar scores at 10 minutes were associated with even higher hazard ratios of cerebral palsy. Compared with children with an Apgar score of 10 at 10 minutes, a 10 minute Apgar score of 3 was associated with a hazard ratio of 425.5 for cerebral palsy, whereas hazard ratios for cerebral palsy in children with Apgar scores of 7, 8, and 9 at 10 minutes were $18.7,9.1$, and 2.4 , respectively.

In total, $3975(0.3 \%)$ children were diagnosed as having epilepsy, corresponding to an incidence rate of 5.1/10 000 child years. Compared with a five minute Apgar score of 10, Apgar scores of 0 and 3 at five minutes were associated with adjusted hazard ratios of 11.9 and 4.4, respectively (fig 2). Hazard ratios of epilepsy decreased with increasing Apgar scores but were significantly increased in offspring with a five minute Apgar score of 7 or less and in offspring with a 10 minute Apgar score of 8 or less. Low Apgar scores were more strongly associated with epilepsy at 10 minutes than at five minutes. The adjusted hazard ratios for epilepsy among children with 10 minute Apgar scores in the 0-3 range had wide 95\% confidence intervals because of small numbers of children in these categories. In children with missing Apgar score at 10 minutes, rates of cerebral palsy and epilepsy were 3.0/10000 child years and 4.2/10 000 child years, respectively (data not shown).

\section{Changes in Apgar scores from five to 10 minutes and risks of cerebral palsy or epilepsy}

Table 1 shows hazard ratios of cerebral palsy in relation to changes in Apgar score from five to 10 minutes. As expected, the highest hazard ratios were seen in offspring with a very low Apgar score at both five and 10 minutes. Increasing Apgar scores from five to 10 minutes were associated with decreasing hazard ratios of cerebral palsy. Compared with children with an Apgar score of 9-10 at both five and 10 minutes, the adjusted hazard ratio for cerebral palsy was 5.3 in children with a score of 7-8 at both five and 10 minutes. Hazard ratios for cerebral palsy were also significantly 


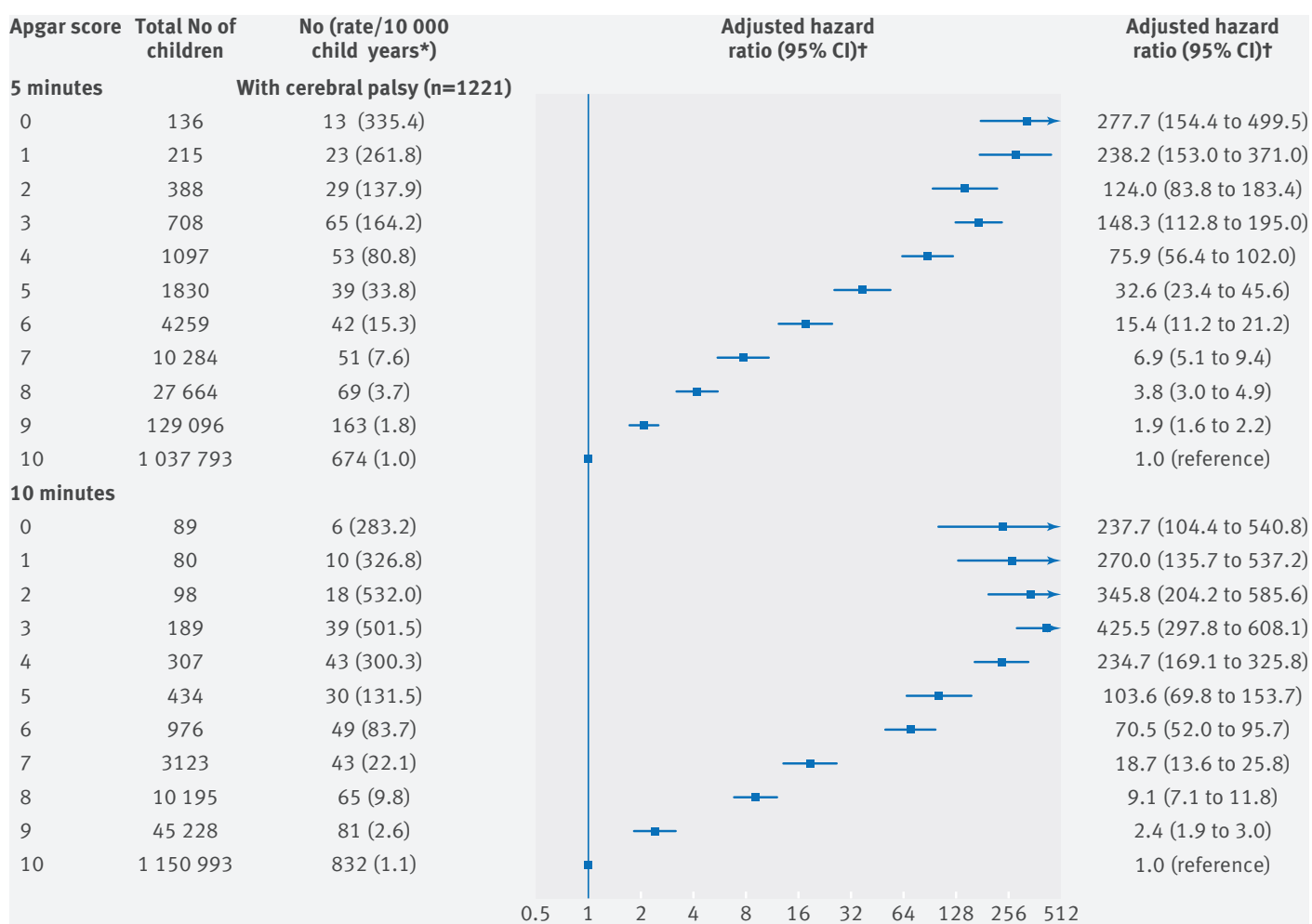

Fig 1 | Apgar score at five and 10 minutes and hazard ratios for cerebral palsy among singleton term live births in Sweden 1999-2012. *Total number of years each child contributed to study. †From multivariable Cox regression models adjusting for maternal factors (smoking, age at child's birth, education, country of birth) and birth characteristics of child (birth order, birth weight for gestational age, gestational age in days, and year of birth)

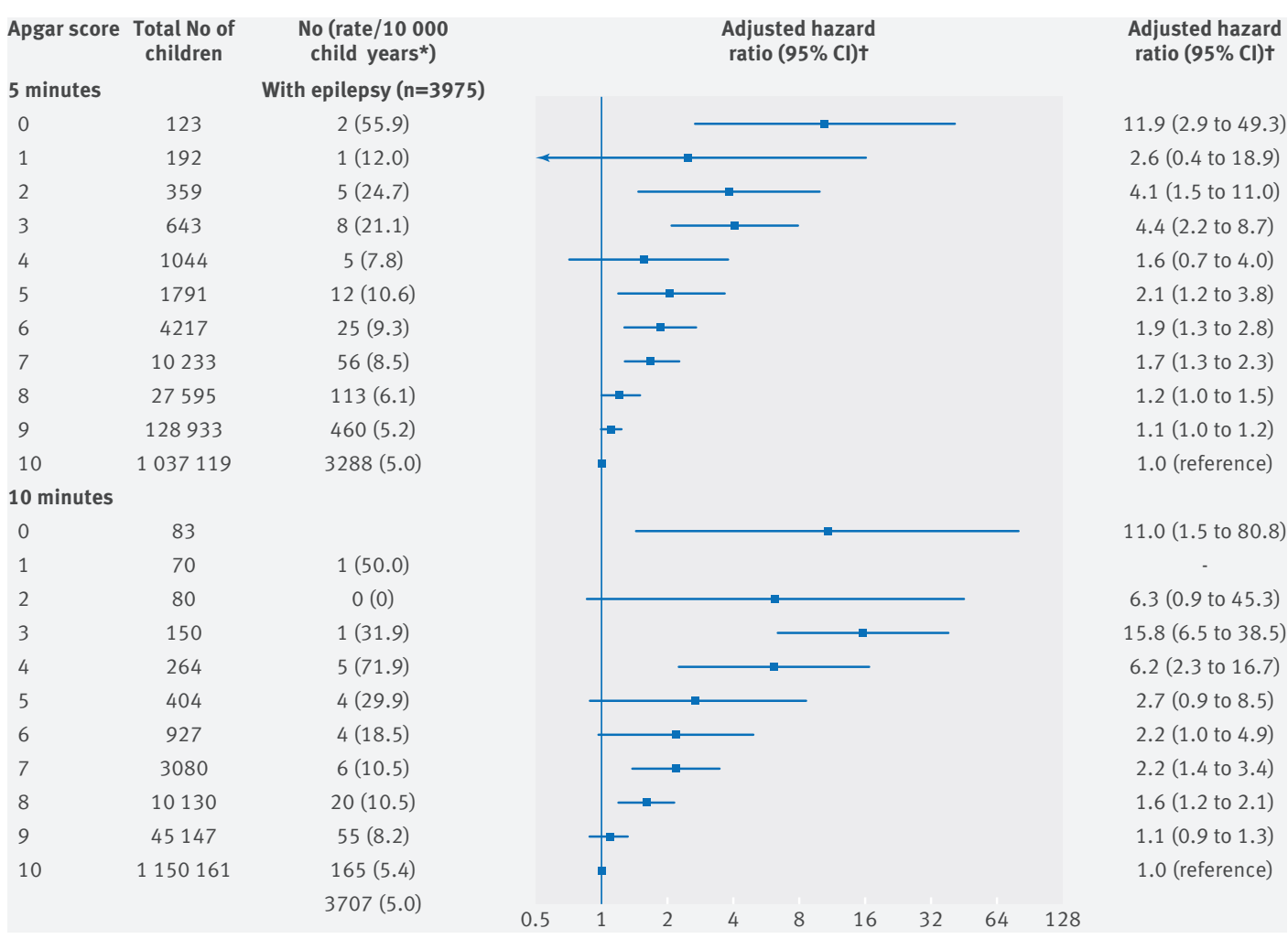

Fig 2 | Apgar score at five and 10 minutes and hazard ratios for epilepsy among singleton term live births in Sweden 1999-2012. *Total number of years each child contributed to study. †From multivariable Cox regression models adjusting for maternal factors (smoking, age at child's birth, education, country of birth, diagnoses of epilepsy) and birth characteristics of child (birth order, birth weight for gestational age, gestational age in days, and year of birth) 
higher among infants with a five minutes Apgar score of 9 and a 10 minute Apgar score of 10 compared with infants who had an Apgar score of 10 at both five and 10 minutes (adjusted hazard ratio 1.3, 95\% confidence interval 1.0 to 1.5 ; table 1 ).

The corresponding adjusted hazard ratios for epilepsy showed less pronounced associations (table 2). Compared with infants with an Apgar score of 9-10 at both five and 10 minutes, the hazard ratio for epilepsy was 1.3 in infants with a five minute score of 7-8 and a 10 minutes score of 9-10, and the adjusted hazard ratio was 1.5 for infants with an Apgar score of 7-8 at both five a 10 minutes. Adjusted hazard ratios for epilepsy were significantly higher among children with a five minute Apgar score of 10 and a 10 minutes score of 9 (table 2).

In supplementary analyses, we additionally adjusted for maternal body mass index and height, which did not substantially change associations between Apgar scores and cerebral palsy or epilepsy (supplementary table B). Although hazard ratios for cerebral palsy were higher following emergency caesarean and operative vaginal delivery (compared with an elective caesarean delivery and spontaneous vaginal delivery), hazard ratios for cerebral palsy increased with decreasing five minute Apgar scores regardless of the mode of delivery (supplementary table C).

\section{Discussion}

This population based cohort study found increasing risks of epilepsy and especially cerebral palsy with decreasing Apgar scores at five and 10 minutes. For both outcomes, the risks associated with a lower Apgar score at 10 minutes were generally higher than those associated with a similar score at five minutes. Notably, an Apgar score of 9 or lower at five or 10 minutes conferred increased risks of cerebral palsy, whereas risks of epilepsy were increased among those with Apgar scores of 7-8 or less at five and 10 minutes. Furthermore, risks of cerebral palsy and epilepsy were increased in offspring with a five minute Apgar score of 7-8, even if the 10 minute Apgar score was 9-10.

Our findings expand on those of previous studies reporting risks of cerebral palsy and epilepsy associated with Apgar scores at one or five minutes. ${ }^{9-12}$ ${ }^{28}$ This study is, to our knowledge, the first to investigate risks of cerebral palsy and epilepsy in relation to Apgar scores at 10 minutes and across the whole range of scores. The increased risks associated with Apgar scores of 8 and 9 are highly noteworthy and worrisome; a 10 minute Apgar score of 8 was associated with a hazard ratio of 9 for cerebral palsy, and a 10 minute Apgar score of 9 was associated with a hazard ratio of 2.4 (fig 1).

\begin{tabular}{|c|c|c|c|c|c|}
\hline 5 min Apgar scores & 10 min Apgar scores & Total No of children & No with outcome & Rate $/ 10000$ child years* & Hazard ratio $(95 \% \mathrm{Cl}) \dagger$ \\
\hline $0-2$ & $0-2$ & 254 & 32 & 422.3 & 222.5 (151.1 to 327.7 ) \\
\hline $0-2$ & $3-4$ & 188 & 26 & 287.7 & 166.8 (110.4 to 252.1$)$ \\
\hline $0-2$ & $5-6$ & 125 & 6 & 81.5 & $41.4(17.1$ to 99.9$)$ \\
\hline $0-2$ & $7-8$ & 101 & 1 & 18.0 & NA \\
\hline $0-2$ & $9-10$ & 56 & 0 & - & - \\
\hline $3-4$ & $0-2$ & 5 & 2 & 1058.2 & 571.7 (205.1 to 1593.4$)$ \\
\hline $3-4$ & $3-4$ & 301 & 56 & 422.6 & $240.2(178.3$ to 323.6$)$ \\
\hline $3-4$ & $5-6$ & 646 & 49 & 130.1 & $76.9(56.7$ to 104.2$)$ \\
\hline $3-4$ & $7-8$ & 654 & 9 & 20.8 & 11.3 (5.6 to 22.8$)$ \\
\hline $3-4$ & $9-10$ & 188 & 2 & 18.4 & $5.2(0.7$ to 37.1$)$ \\
\hline $5-6$ & $0-2$ & 8 & 0 & - & - \\
\hline $5-6$ & $3-4$ & 7 & 0 & - & - \\
\hline $5-6$ & $5-6$ & 639 & 24 & 66.1 & 34.8 (22.4 to 53.9) \\
\hline $5-6$ & $7-8$ & 3324 & 50 & 23.3 & $15.3(11.5$ to 20.4$)$ \\
\hline $5-6$ & $9-10$ & 2065 & 7 & 5.2 & $3.2(1.5$ to 6.7$)$ \\
\hline $7-8$ & $0-2$ & 0 & 0 & - & - \\
\hline $7-8$ & $3-4$ & 0 & 0 & - & - \\
\hline $7-8$ & $5-6$ & 0 & 0 & - & - \\
\hline $7-8$ & $7-8$ & 8779 & 48 & 8.4 & $5.3(3.9$ to 7.1$)$ \\
\hline $7-8$ & $9-10$ & 28889 & 71 & 3.6 & 2.2 (1.8 to 2.9$)$ \\
\hline $9-10$ & $0-2$ & 0 & 0 & - & - \\
\hline $9-10$ & $3-4$ & 0 & 0 & - & - \\
\hline $9-10$ & $5-6$ & 0 & 0 & - & - \\
\hline $9-10$ & $7-8$ & 460 & 0 & - & - \\
\hline $9-10$ & $9-10$ & 1165023 & 833 & 1.1 & 1.0 (reference) $\ddagger$ \\
\hline 9 & 9 & 28939 & 31 & 1.6 & $1.0(0.7$ to 1.5$)$ \\
\hline 9 & 10 & 99385 & 129 & 1.9 & 1.3 (1.0 to 1.5$)$ \\
\hline 10 & 9 & 513 & 1 & 3.3 & NA \\
\hline 10 & 10 & 1036186 & 672 & 1.0 & 1.0 (reference)§ \\
\hline \multicolumn{6}{|c|}{$\begin{array}{l}\text { NA=hazard rations could not be reliably calculated because of small number of cases. } \\
{ }^{*} \text { Total number of years each child contributed to study. } \\
\text { †Adjusted for maternal factors (smoking, age at child's birth, education, and country of birth) and birth characteristics of child (birth order, birth weight for gestational age, gestational age in days, } \\
\text { and year of birth). } \\
\text { †Reference category for all Apgar values in rows above. } \\
\text { §Reference category for Apgar values in three rows above. }\end{array}$} \\
\hline
\end{tabular}




\begin{tabular}{|c|c|c|c|c|c|}
\hline 5 min Apgar scores & 10 min Apgar scores & Total No of children & No with outcome & Rate/10 000 child years* & Hazard ratio $(95 \% \mathrm{Cl}) \dagger$ \\
\hline $0-2$ & $0-2$ & 254 & 2 & 28.5 & $6.4(1.6$ to 25.8$)$ \\
\hline $0-2$ & $3-4$ & 188 & 4 & 47.7 & $8.5(2.7$ to 26.7$)$ \\
\hline $0-2$ & $5-6$ & 125 & 1 & 14.0 & NA \\
\hline $0-2$ & $7-8$ & 101 & 1 & 18.3 & NA \\
\hline $0-2$ & $9-10$ & 56 & 0 & - & - \\
\hline $3-4$ & $0-2$ & 5 & 0 & - & - \\
\hline $3-4$ & $3-4$ & 301 & 5 & 43.4 & $7.9(2.9$ to 21.1$)$ \\
\hline $3-4$ & $5-6$ & 646 & 4 & 11.0 & $2.5(0.9$ to 6.5$)$ \\
\hline $3-4$ & $7-8$ & 654 & 4 & 9.4 & $2.1(0.8$ to 5.6$)$ \\
\hline $3-4$ & $9-10$ & 188 & 0 & - & - \\
\hline $5-6$ & $0-2$ & 8 & 0 & - & - \\
\hline $5-6$ & $3-4$ & 7 & 0 & - & - \\
\hline $5-6$ & $5-6$ & 639 & 5 & 14.2 & 2.5 (1.0 to 6.8$)$ \\
\hline $5-6$ & $7-8$ & 3324 & 23 & 11.0 & $2.2(1.4$ to 3.4$)$ \\
\hline $5-6$ & $9-10$ & 2065 & 9 & 6.7 & $1.0(0.4$ to 2.2$)$ \\
\hline $7-8$ & $0-2$ & 0 & 0 & - & - \\
\hline $7-8$ & $3-4$ & 0 & 0 & - & - \\
\hline $7-8$ & $5-6$ & 0 & 0 & - & - \\
\hline 7-8 & $7-8$ & 8779 & 44 & 7.9 & 1.5 (1.0 to 2.0$)$ \\
\hline $7-8$ & $9-10$ & 28889 & 125 & 6.5 & $1.3(1.1$ to 1.6$)$ \\
\hline $9-10$ & $0-2$ & 0 & 0 & - & - \\
\hline 9-10 & $3-4$ & 0 & 0 & - & - \\
\hline $9-10$ & $5-6$ & 0 & 0 & - & - \\
\hline $9-10$ & $7-8$ & 460 & 3 & 10.9 & NA \\
\hline $9-10$ & $9-10$ & 1165023 & 3738 & 5.0 & 1.0 (reference) $\ddagger$ \\
\hline 9 & 9 & 28908 & 93 & 4.7 & 0.9 (0.8 to 1.2$)$ \\
\hline 9 & 10 & 99256 & 361 & 5.3 & 1.1 (1.0 to 1.2$)$ \\
\hline 10 & 9 & 512 & 4 & 13.3 & 2.7 (1.1 to 7.2$)$ \\
\hline 10 & 10 & 1035514 & 3280 & 5.0 & 1.0 (reference)§ \\
\hline \multicolumn{6}{|c|}{$\begin{array}{l}\text { NA=hazard rations could not be reliably calculated because of small number of cases. } \\
\text { *Total number of years each child contributed to the study. } \\
\text { †Adjusted for maternal factors (smoking, age at child’s birth, education, country of birth, and diagnoses of epilepsy) and birth characteristics of child (birth order, birth weight for gestational age, } \\
\text { gestational age in days, and year of birth). } \\
\text { fReference category for all Apgar values in rows above. } \\
\text { §Reference category for Apgar values in three rows above. }\end{array}$} \\
\hline
\end{tabular}

Our study also quantifies the risks of cerebral palsy and epilepsy associated with changes between five and 10 minute Apgar scores. The finding that children with five minute Apgar scores of 7-8 and 10 minute Apgar scores of 7-8 have higher hazard ratios of cerebral palsy and epilepsy (hazard ratios were 5.3 and 1.5, respectively) is concerning and warrants critical attention from the resuscitation community.

\section{Study strengths and limitations}

The primary strengths of the study are the population based design and its large sample size, which enabled quantification of the risks of cerebral palsy and epilepsy across the whole range of Apgar scores and in relation to changes in Apgar score from five to 10 minutes. Data on both exposures and outcomes were collected prospectively, and diagnoses of cerebral palsy and epilepsy were made independently of Apgar scores, limiting risk of bias. By using data from several national registries, we identified the vast majority of individuals with cerebral palsy and epilepsy. Furthermore, we were able to adjust for important confounders. In Sweden, all citizens have free access to uniform publicly funded healthcare, which contributes to high internal validity. We used a validated definition of epilepsy, ${ }^{20}$ and cerebral palsy was typically diagnosed according to the Surveillance of Cerebral Palsy in Europe (SCPE) guidelines used by the Swedish National Quality Registry (CPUP). ${ }^{24}$

However, our study also had some limitations. Cerebral palsy and epilepsy are heterogeneous conditions with varying causes, clinical presentation, severity, and prognosis. We did not investigate the effect of Apgar scores on risks of subtypes of cerebral palsy or epilepsy. The study period spanned 13 years, and advances in obstetric and neonatal care over this time may have influenced outcomes. Induced hypothermia in term infants with hypoxic ischaemic encephalopathy may reduce risks of death and neurodevelopmental disorders. ${ }^{29-31}$ This treatment was introduced in Sweden in 2007, national coverage began in 2010, and the therapy is now strongly recommended by the Swedish National Society for term infants with perinatal asphyxia. However, we did not have information on obstetric and neonatal interventions and were unable to explore the effect of this treatment. Nevertheless, our analyses adjusted for year of birth, and this would have potentially overcome the effects of temporal changes in neonatal care. Lastly, we cannot rule out possible influence of other unmeasured or unknown factors.

\section{Potential mechanisms}

Cerebral palsy and childhood onset epilepsy are serious neurological disorders associated with increased 
risks of morbidity and mortality. ${ }^{25} 26$ The causes of cerebral palsy and epilepsy are multifactorial, 2527 32-35 but prenatal and perinatal events are important risk factors for both conditions. Cerebral palsy is commonly referred to as an umbrella diagnosis with reference to cause, severity, and symptoms. Awareness of the importance of an early diagnosis of cerebral palsy is increasing given that cerebral palsy can often be diagnosed before 5 months of age. Early identification of cerebral palsy and active intervention may alter neuroplasticity and optimise the child's psychomotor development. $^{36}$ Seventy per cent of children with cerebral palsy are born at term, and the cause of cerebral palsy differs between preterm and term infants. Data from animal studies suggest that brain injury secondary to ischaemia/hypoxia is a more prevalent cause of cerebral palsy in term children than in preterm children. ${ }^{25}$ Hypoxia may lead to energy depletion, oxidative stress, and inflammation, ultimately resulting in cell death. ${ }^{25}$ However, a comprehensive review by Ellenberg and Nelson states that only a small proportion of all cases of cerebral palsy are due to perinatal asphyxia. ${ }^{37}$ Reduced vitality in term born infants has a variety of potential causes with clinically similar manifestations. For example, maternal fever and inflammatory conditions are common antecedents of low Apgar scores, depressed respiration, and other symptoms that are also common in hypoxia. Thus, other adverse prenatal or perinatal events, equally deleterious to the developing brain, including inflammation in the maternal/fetal compartments, ${ }^{38}$ may have contributed to our findings. ${ }^{37}$ Nevertheless, in our study of children born at term, perinatal hypoxia may be one of the underlying factors that contributed to the increased risks of cerebral palsy among infants with lower Apgar scores.

Several risks for epilepsy have been identified, including both maternal and perinatal factors. ${ }^{35}$ 39 We have previously shown increased risks of epilepsy in newborns with neonatal infections, hyperbilirubinaemia, hypoglycaemia, and respiratory disorders. ${ }^{39}$ These conditions are more frequent in infants with low Apgar scores. Results of the current study are in line with previous reports, showing elevated risks of epilepsy in offspring with decreased Apgar scores at one or five minutes after birth. ${ }^{911}$ Our findings of higher risks of epilepsy with low Apgar scores at 10 minutes (compared with low Apgar scores at five minutes), and also the findings of higher risks associated with changes in five and 10 minute Apgar scores within the normal range, may facilitate identification of infants at high risk.

Maternal obesity has previously been associated with increased risks of low Apgar score, cerebral palsy, and epilepsy. ${ }^{39-41}$ In this study, adjusting for maternal body mass index in early pregnancy did not substantially change risks, indicating that maternal obesity does not modify the relation between Apgar scores and severe neurological disorders in children.

Mode of delivery may affect Apgar scores. We found that risks of cerebral palsy associated with
Apgar scores differed by mode of delivery, and the highest risks were observed for offspring delivered by emergency caesarean section. A more detailed analysis of the underlying reasons behind this finding was not possible, as we did not have information on indications for elective or emergency caesarean sections.

\section{Conclusion}

We found increasing risks of epilepsy and especially cerebral palsy with decreasing Apgar scores at five and 10 minutes in offspring born at term. Importantly, even a slight decrease in Apgar scores at five or 10 minutes (scores of 8 or 9 for cerebral palsy and scores of 7 or 8 for epilepsy) increased risks of cerebral palsy and epilepsy. Although an increase in Apgar score values from five to 10 minutes improved outcome, the risks of cerebral palsy and epilepsy remained higher among children with an Apgar score of 9-10 at 10 minutes if their five minute Apgar score was 7-8. Even an Apgar score of 9 at five minutes and a 10 minute score of 10 was associated with a slightly increased hazard ratio for cerebral palsy, and the hazard ratio for epilepsy was increased in infants with a full score at five minutes and a 10 minute score of 9 . This is of particular interest as in many settings, neonatologists, midwifes, and other care providers will assign the newborn a 10 minute Apgar score only when a low five minute Apgar score is noted. We believe that our findings are widely applicable and provide justification for assigning all newborns an Apgar score at one, five, and 10 minutes and continuing active neonatal resuscitation of infants who are mildly compromised at five minutes.

Contributors: MP and NR contributed equally to this paper. NR, SC, KSJ, and KT conceived and designed the study. All authors acquired, analysed, and interpreted the data and critically revised the manuscript for important intellectual content. MP drafted the manuscript. NR did the statistical analysis. SC obtained funding and provided administrative, technical, or material support. NR and SC are the guarantors.

Funding: This study was funded by the Swedish Research Council for Health, Working Life and Welfare (grant No 2014-0073), by the Stockholm County Council (ALF project 20150118 and a clinical postdoc position to MP), and by an unrestricted grant from Karolinska Institutet (No 2368/10-221, distinguished professor award to SC). NR is supported by a postdoctoral fellowship award from the Canadian Institutes of Health Research (CIHR). KS) is supported by the BC Children's Hospital Research Institute and a chair award from the CIHR (APR-126338). Funders were not involved in the design and conduct of the study; collection, management, analysis, or interpretation of the data; or preparation, review, or approval of the manuscript.

Competing interests: All authors have completed the ICMJE uniform disclosure form at www.icmje.org/coi_disclosure.pdf and declare: no support from any organisation for the submitted work other than that described above; no financial relationships with any organisations that might have an interest in the submitted work in the previous three years; no other relationships or activities that could appear to have influenced the submitted work.

Ethical approval: The study was approved by the Regional Ethic Review Board at Karolinska Institutet, Stockholm, Sweden (No 2011/195-31/2)

Data sharing: No additional data available.

Transparency: The lead authors (MP and NR) affirm that the manuscript is an honest, accurate, and transparent account of the study being reported; that no important aspects of the study have been omitted; and that any discrepancies from the study as planned (and, if relevant, registered) have been explained.

This is an Open Access article distributed in accordance with the Creative Commons Attribution Non Commercial (CC BY-NC 4.0) license, 
which permits others to distribute, remix, adapt, build upon this work non-commercially, and license their derivative works on different terms, provided the original work is properly cited and the use is noncommercial. See: http://creativecommons.org/licenses/by-nc/4.0/.

1 Apgar V. A proposal for a new method of evaluation of the newborn infant. Curr Res Anesth Analg 1953;32:260-7. doi:10.1213/00000539-195301000-00041

2 Berglund S, Pettersson $\mathrm{H}$, Cnattingius S, et al. How often is a low Apgar score the result of substandard care during labour? BJOG 2010;117:968-78doi:10.1111/j.1471-0528.2010.02565.x.

3 Lai S, Flatley C, Kumar S. Perinatal risk factors for low and moderate five-minute Apgar scores at term. Eur J Obstet Gynecol Reprod Biol 2017;210:251-6. doi:10.1016/j.ejogrb.2017.01.008

4 Hogan L, Ingemarsson I, Thorngren-Jerneck K, Herbst A. How often is a low 5-min Apgar score in term newborns due to asphyxia? Eur J Obstet Gynecol Reprod Biol 2007;130:169-75. doi:10.1016/j. ejogrb.2006.03.002

5 Catlin EA, Carpenter MW, Brann BS 4th, et al. The Apgar score revisited: influence of gestational age. J Pediatr 1986;109:865-8. doi:10.1016/S0022-3476(86)80715-6

6 Hegyi T, Carbone T, Anwar M, et al. The apgar score and its components in the preterm infant. Pediatrics 1998;101:77-81. doi:10.1542/peds.101.1.77

7 Wong L, MacLennan AH. Gathering the evidence: cord gases and placental histology for births with low Apgar scores. Aust N Z J Obstet Gynaecol 2011;51:17-21. doi:10.1111/j.1479828X.2010.01275.x

8 Rouse DJ, Landon M, Leveno KJ, et al, National Institute of Child Health And Human Development, Maternal-Fetal Medicine Unit Network. The Maternal-Fetal Medicine Units cesarean registry: chorioamnionitis at term and its duration-relationship to outcomes. Am J Obstet Gynecol 2004;191:211-6. doi:10.1016/j.ajog.2004. 03.003

9 Thorngren-Jerneck K, Herbst A. Low 5-minute Apgar score: a population-based register study of 1 million term births. Obstet Gynecol 2001;98:65-70.

10 Moster D, Lie RT, Irgens LM, Bjerkedal T, Markestad T. The association of Apgar score with subsequent death and cerebral palsy: A population-based study in term infants. J Pediatr 2001;138: 798-803. doi:10.1067/mpd.2001.114694

11 Sun Y, Vestergaard M, Pedersen CB, Christensen J, Olsen J. Apgar scores and long-term risk of epilepsy. Epidemiology 2006;17: 296-301. doi:10.1097/01.ede.0000208478.47401.b6

12 Lie KK, Grøholt EK, Eskild A. Association of cerebral palsy with Apgar score in low and normal birthweight infants: population based cohort study. BMJ 2010;341:c4990. doi:10.1136/bmj.c4990

13 Nelson KB, Ellenberg JH. Apgar scores as predictors of chronic neurologic disability. Pediatrics 1981;68:36-44.

14 Ludvigsson JF, Almqvist C, Bonamy AK, et al. Registers of the Swedish total population and their use in medical research. Eur J Epidemiol 2016;31:125-36. doi:10.1007/s10654-016-0117-y

15 Swedish National Board of Health and Welfare. The Swedish Medical Birth Register: a summary of content and quality. 2003. https://www.socialstyrelsen.se/Lists/Artikelkatalog/ Attachments/10655/2003-112-3 20031123.pdf.

16 Ludvigsson JF, Andersson E, Ekbom A, et al. External review and validation of the Swedish national inpatient register. BMC Public Health 2011;11:450. doi:10.1186/1471-2458-11-450

17 Swedish National Board of Health and Welfare. Quality and content in the Swedish Patient Register. 2009. http://www.socialstyrelsen.se/Lists/Artikelkatalog/ Attachments/8306/2009-125-15 200912515 rev2.pdf.

18 Statistics Sweden. Evaluation of the Swedish register of education. 2006. http://www.scb.se/statistik/_publikationer/ BE9999 2006A01 BR_BE96ST0604.pdf.

19 Helmers SL, Thurman DJ, Durgin TL, Pai AK, Faught E. Descriptive epidemiology of epilepsy in the U.S. population: A different approach. Epilepsia 2015;56:942-8. doi:10.1111/epi.13001

20 Thurman DJ, Beghi E, Begley CE, et al, ILAE Commission on Epidemiology. Standards for epidemiologic studies and surveillance of epilepsy. Epilepsia 2011;52(Suppl 7):2-26. doi:10.1111/j.15281167.2011.03121.x

21 World Health Organization. Global Database on Body Mass Index: BMI classification. http://apps.who.int/bmi/index.jsp.

22 Marsál K, Persson PH, Larsen T, Lilja H, Selbing A, Sultan B. Intrauterine growth curves based on ultrasonically estimated foetal weights. Acta Paediatr 1996;85:843-8. doi:10.1111/j.1651-2227.1996.tb14164.x

23 Eun S, Lee IM, Yi DY, et al. Assessment of the association between Apgar scores and seizures in infants less than 1 year old. Seizure 2016;37:48-54. doi:10.1016/j.seizure.2016.03.001

24 Surveillance of Cerebral Palsy in Europe. Surveillance of cerebral palsy in Europe: a collaboration of cerebral palsy surveys and registers. Dev Med Child Neurol 2000;42:816-24.

25 Graham HK, Rosenbaum P, Paneth N, et al. Cerebral palsy. Nat Rev Dis Primers 2016:2:15082. doi:10.1038/nrdp.2015.82

26 Shankar R, Donner EJ, McLean B, Nashef L, Tomson T. Sudden unexpected death in epilepsy (SUDEP): what every neurologist should know. Epileptic Disord 2017;19:1-9.

27 Guerrini R. Epilepsy in children. Lancet 2006;367:499-524. doi:10.1016/S0140-6736(06)68182-8

28 Thorngren-Jerneck K, Herbst A. Perinatal factors associated with cerebral palsy in children born in Sweden. Obstet Gynecol 2006;108:1499-505. doi:10.1097/01.AOG.0000247174. $27979.6 b$

29 Laptook AR, Shankaran S, Tyson JE, et al, Eunice Kennedy Shriver National Institute of Child Health and Human Development Neonatal Research Network. Effect of Therapeutic Hypothermia Initiated After 6 Hours of Age on Death or Disability Among Newborns With Hypoxic-Ischemic Encephalopathy: A Randomized Clinical Trial. JAMA 2017;318:1550-60. doi:10.1001/jama.2017.14972

30 Gluckman PD, Wyatt JS, Azzopardi D, et al. Selective head cooling with mild systemic hypothermia after neonatal encephalopathy: multicentre randomised trial. Lancet 2005;365:663-70. doi:10.1016/S0140-6736(05)17946-X

31 Jacobs SE, Berg M, Hunt R, Tarnow-Mordi WO, Inder TE, Davis PG Cooling for newborns with hypoxic ischaemic encephalopathy. Cochrane Database Syst Rev 2013;(1):CD003311.

32 Whitehead E, Dodds L, Joseph KS, et al. Relation of pregnancy and neonatal factors to subsequent development of childhood epilepsy: a population-based cohort study. Pediatrics 2006;117:1298-306. doi:10.1542/peds.2005-1660

33 Painter B. Neurology. 3rd ed. Saunders, 1998: 717-23.

34 Odding E, Roebroeck ME, Stam HJ. The epidemiology of cerebral palsy: incidence, impairments and risk factors. Disabil Rehabil 2006:28:183-91 doi:10.1080/09638280500158422

35 Walsh S, Donnan J, Fortin Y, et al. A systematic review of the risks factors associated with the onset and natural progression of epilepsy. Neurotoxicology 2017;61:64-77. doi:10.1016/j.neuro.2016.03.011

36 Novak I, Morgan C, Adde L, et al. Early, Accurate Diagnosis and Early Intervention in Cerebral Palsy: Advances in Diagnosis and Treatment. JAMA Pediatr 2017;171:897-907. doi:10.1001/ jamapediatrics.2017.1689

37 Ellenberg JH, Nelson KB. The association of cerebral palsy with birth asphyxia: a definitional quagmire. Dev Med Child Neurol 2013:55:210-6. doi:10.1111/dmcn.12016

38 Dan B, Mayston M, Paneth N, Rosenbloom L, eds. Cerebral palsy: science and clinical practice. Mac Keith Press, 2014

39 Razaz N, Tedroff K, Villamor E, Cnattingius S. Maternal Body Mass Index in Early Pregnancy and Risk of Epilepsy in Offspring. JAMA Neurol 2017;74:668-76. doi:10.1001/jamaneurol.2016.6130

40 Persson M, Johansson S, Villamor E, Cnattingius S. Maternal overweight and obesity and risks of severe birth-asphyxia-related complications in term infants: a population-based cohort study in Sweden. PLoS Med 2014;11:e1001648. doi:10.1371/journal. pmed.1001648

41 Villamor E, Tedroff K, Peterson M, et al. Association Between Maternal Body Mass Index in Early Pregnancy and Incidence of Cerebral Palsy. JAMA 2017:317:925-36. doi:10.1001/jama.2017.0945

Supplementary: tables 